Am. J. Trop. Med. Hyg., 64(1, 2), 2001, pp. 32-34

Copyright $(\mathcal{C} 2001$ by The American Society of Tropical Medicine and Hygiene

\title{
COMPARISON OF DIRECT AND MEMBRANE FEEDING METHODS TO INFECT ANOPHELES ARABIENSIS WITH PLASMODIUM FALCIPARUM
}

\author{
HERMAN P. AWONO-AMBENE, LAMINE, DIAWARA, AND VINCENT ROBERT \\ Laboratoire de Paludologie, Institut de Recherche pour le Développement, Dakar, Sénégal; Université Cheikh Anta Diop, \\ Dakar, Sénégal; Service de Lutte Anti-Parasitaire, Thiès, Sénégal
}

\begin{abstract}
Two standard methods are available to infect mosquitoes with malaria parasites: direct feeding through the skin of the gametocyte carrier, and membrane feeding. Anopheles arabiensis collected at larval stages and reared in an insectary were fed in parallel by feeding on Plasmodium falciparum gametocyte carriers and by membrane feeding on venous blood of the same gametocyte carriers. Infection of mosquitoes was assessed at Day 7 post bloodmeal by oocyst count of the mosquito midguts. The following parameters were not significantly different between the two methods: the percentage of gametocyte carriers infective for at least one mosquito (52.4\% through the skin versus $57.1 \%$ through the membrane), the mean infection rate of mosquitoes (10.0\% versus $11.3 \%)$, the geometric mean oocyst number per mosquito ( 2.51 versus 3.83 ). In conclusion, infection of mosquitoes by membrane feeding was similar to infection by direct feeding. Most of the volunteers preferred venipuncture to mosquito bites.
\end{abstract}

\section{INTRODUCTION}

Infection of mosquitoes by Plasmodium occurs by the ingestion of gametocytes during a bloodmeal. Two methods are available to perform this infection: first, a natural bloodmeal taken by the mosquito through the skin of a gametocyte carrier and second, a venipuncture followed by ingestion of this blood by the mosquito through the membrane of a feeder apparatus. ${ }^{1}$ These two methods differ in several ways. Ingested blood comes from capillaries for direct feeding but from veins for membrane feeding. In the membrane feeding method, an anticoagulant is added and blood is handled before being put in the feeder. Furthermore, during the course of the latter, blood undergoes chemical changes and its cellular components settle. It is not known whether or how these differences affect infectivity for the mosquito. This study compared the infection of Anopheles arabiensis by Plasmodium falciparum gametocytes by direct feeding through the skin and by feeding through a membrane. The maintenance and handling of mosquitoes were previously standardized. ${ }^{2,3}$

\section{MATERIALS AND METHODS}

The study was performed at Thiès $\left(14^{\circ} 47^{\prime} \mathrm{N}, 16^{\circ} 55^{\prime} \mathrm{E}\right)$, Sénégal, a town located $70 \mathrm{~km}$ east of Dakar. This is an endemic area in which malaria transmission is highly seasonal. The study was conducted between September and December 1998 when malaria cases were most frequent.

Gametocyte carriers. Plasmodium falciparum gametocyte carriers were identified among residents in the area of Thiès. Persons $>5$ years old (or their parents) were informed of the protocol in which their participation was requested. Those who consented and did not harbor other Plasmodium species were included in the study. The protocol was approved by the Ministry of Health of Senegal.

Mosquitoes. Anopheles arabiensis were collected at larval stages in breeding sites (pools and wells) in Dakar. ${ }^{4}$ The larvae were reared in the Dakar insectary at $27-28^{\circ} \mathrm{C}$. Adults were maintained at the same temperature and at $70-90 \%$ relative humidity, with a continuously available $5 \%$ sucrose solution. No bloodmeal was ingested before infection. Three-day-old females were placed without males in a paper cup covered with a mosquito net (volume $=60 \mathrm{ml}$; diameter $=6 \mathrm{~cm}$; height $=2.5 \mathrm{~cm}$ ) and starved for 5-6 hours before the bloodmeal. Mosquitoes were transported in a box isolated from light and heat under a dampened floor cloth for the approximately $1.5 \mathrm{hr}$ road trip to Thiès. The temperatures inside the box varied from $25.5-31.0^{\circ} \mathrm{C}$ with a mean difference between the minimum and the maximum of $1.32^{\circ} \mathrm{C}$.

Blood handling and mosquito engorgement. Venipuncture at the antecubital fossa was performed on the human gametocyte carriers between 10 AM and 1 PM and the blood collected into a sterile lithium heparinate Vacutainer ${ }^{\circledR}$ tube (Becton Dickinson, Franklin Lakes, NJ). A membrane feeder (diameter $2.5 \mathrm{~cm}$, surface area $5.0 \mathrm{~cm}^{2}$ as described by Ponnudurai and others $)^{5}$ held at $37.5^{\circ} \mathrm{C}$ was immediately filled with $1.5 \mathrm{ml}$ of blood using a sterile syringe. Mosquitoes were allowed to feed through a Baudruche membrane (Joseph Long Inc., Belleville, NJ) for 10 minutes in a dark place. Eighty female mosquitoes in 2 cups of 40 mosquitoes each were used, the first cup being placed under the membrane for ten minutes then a second cup under the same membrane for the next 10 minutes. After engorgement, the feeder and the Baudruche membrane were cleaned by rinsing in a large amount of water without detergent and immersed in $95 \%$ ethanol and dried before reuse.

The direct feeding was started at the same time as the membrane feeding. It involved two cups of 35 female mosquitoes each simultaneously placed in contact with the calves of the gametocyte carrier for 15 minutes in the dark. After direct feeding, a painkilling ointment was applied.

Blood remaining in the blood collection set was used to make thick blood films. They were Giemsa-stained and 200 microscopic oil-immersion fields were examined. The gametocyte density was estimated on the basis of 8,000 leukocytes $/ \mathrm{mm}^{3}$ blood.

Treatment of mosquitoes. After engorgement, mosquitoes were transported by road to the Dakar insectary. Mosquitoes were individually examined and those not fully engorged were removed. Fully fed mosquitoes (no more than 15 per cup) were maintained in paper cups at $26-28^{\circ} \mathrm{C}$ and $70-90 \%$ relative humidity without any further bloodmeals. Cotton wool impregnated with sucrose solution was put on each cup and replaced twice a day. Seven days after the bloodmeal, surviving mosquitoes were dissected for microscopic examination of the oocysts in the midgut using one drop of $1 \%$ mercurochrome. 


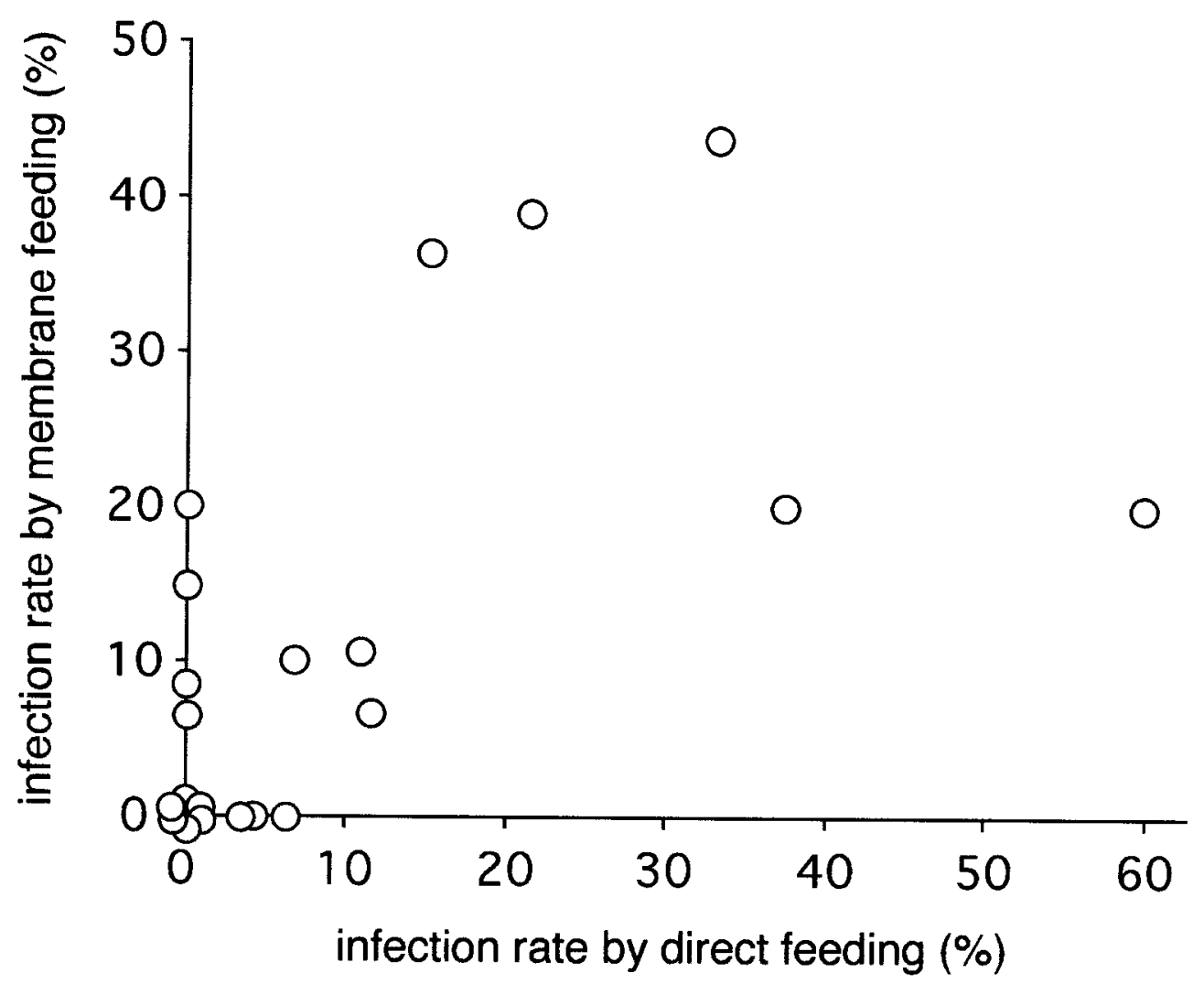

FIGURE 1. Relationship between infection rates of Anopheles arabiensis fed with the blood of 21 Plasmodium falciparum gametocyte carriers using two methods. The 3 circles on the horizontal axis designate gametocyte carriers who infected mosquitoes only by direct feeding. The 4 circles on the vertical axis indicate mosquitoes infected only by membrane feeding. The 8 circles in the central part of the figure, mosquitoes infected by both methods. The 6 circles on the origin of the two axis, mosquitoes not infected by the two methods.

Data treatment and statistical analysis. The blood-feeding rate is the proportion of female mosquitoes which were fully fed after a bloodmeal. The survival rate is the proportion of engorged females which survived 7 days after the bloodmeal. The infection rate is the proportion of mosquitoes which had one or more oocysts. The arithmetic mean observed in various experiments was calculated. The geometric mean of Williams [ $=$ exponential (arithmetic mean $\left.\left.\left(\ln \left(x_{i}+1\right)\right)\right)-1\right]$ was used to calculate oocyst mean number to allow the use of zeros. ${ }^{6}$

Calculations and infection rates were only performed for experiments in which at least 10 mosquito midguts were examined by the two methods. The means of paired series were compared using the non-parametric rank sum test of Wilcoxon. Fisher's exact probability test was used to compare the proportions of gametocyte carriers infective for mosquitoes.

\section{RESULTS}

Data were obtained using $60 P$. falciparum gametocyte carriers and 9,000 An. arabiensis mosquitoes of which 4,335 were fully engorged. The mean blood-feeding rate was $65.2 \%$ after direct feeding versus $32.7 \%$ after membrane feeding ( $P<0.0001$ by Wilcoxon test, $\mathrm{n}=60$ pairs). The mean survival rate seven days after engorgement was $34.3 \%$ after direct feeding versus $28.1 \%$ after membrane feeding $(P$ $=0.006, \mathrm{n}=57$ pairs).

Comparison of the direct feeding method to the membrane feeding method in terms of mosquito infectivity was made on 21 gametocyte carriers. The mean age of the gametocyte carriers was 20.3 years (range, 7-48; median, 17). Eighteen carriers had already received antimalarial treatment (12 with quinine, 4 with chloroquine, 2 with quinine plus chloroquine or sulfadoxine-pyrimethamine). Five had fever (body temperature $>37.5^{\circ} \mathrm{C}$ ) at the time the mosquitoes were taking a bloodmeal. Twenty mentioned a recent malaria attack and $6 \mathrm{had}$ an asexual parasitemia (geometric mean $=166 / \mu \mathrm{L}$; maximum, 1,310/ $\mu \mathrm{L}$ ). The geometric mean gametocytemia was $214 / \mu \mathrm{L}$ blood (range $=6-1,369$ ). The proportion of gametocyte carriers who infected at least one mosquito was $71 \%(15 / 21)$. This value was $52.4 \%(11 / 21)$ after direct feeding versus $57.1 \%(12 / 21)$ after membrane feeding $(P=$ 0.77). The degree of concordance between the two methods was $66.7 \%(14 / 21)$. The difference between the discordant pairs $(4 / 21$ versus $3 / 21)$ was not significant $(P=0.70)$. The mean infection rate was $10.0 \%$ after direct feeding versus $11.3 \%$ after membrane feeding $(P=0.43$ by Wilcoxon test $)$ with a maximum of $60 \%$ after direct feeding and $44 \%$ after membrane feeding (Figure 1). A strong correlation existed between direct and membrane feeding $(r=0.605, \mathrm{n}=21$, $P=0.0029$ ). The maximum number of oocysts observed on one midgut was 60 after direct feeding versus 252 after membrane feeding. The geometric mean number of oocysts per mosquito was 2.51 after direct feeding versus 3.83 after membrane feeding $(P=0.16)$. A strong correlation existed 
for the geometric means of direct and membrane feeding $(r$ $=0.869, \mathrm{n}=21, P<0.0001)$.

The carrier's age, body temperature, recent antimalarial treatment, and asexual parasitemia were not linked to the infection rate of mosquitoes or the mean number of oocysts. When fifty of the volunteer gametocyte carriers were asked whether they preferred to provide blood by venipuncture or by the direct feeding method, $94 \%$ preferred the former and $6 \%$ the latter.

\section{DISCUSSION}

This study demonstrates concordance between two widely used methods to infect anopheline mosquitoes with $P$. falciparum gametocytes. The results have two implications: first, studies of experimental infections performed by membrane feeding ${ }^{7-10}$ and by direct feeding ${ }^{11-13}$ have epidemiological relevance and second, trials to evaluate the efficacy of a transmission-blocking vaccine can use either method. Volunteers appear to favor providing blood by venipuncture for the membrane feeding method rather than directly to mosquitoes feeding on their skins.

The overall significance of the current study is limited by two factors. First, blood-feeding and survival rates were relatively weak. This was mainly due to the use of wild vectors who had not been selected for maintenance and survival in the environmental conditions in the insectary. This use of wild-caught vector material along with naturally-infected humans provide data that reflect both the infectiousness of the parasite and the susceptibility of its local vector in the Dakar area. After several generations in an insectary, the ability of mosquitoes to be infected with Plasmodium may be altered compared to the original wild generation. ${ }^{14}$ Second, the infection rate of mosquitoes was also relatively low. In our study, the mean percentage of infected mosquitoes was $11 \%$, which is slightly lower than found in comparable reports (12\% in the Bobo-Dioulasso area of Burkina Faso; ${ }^{7} 19 \%$ in Yaounde, Cameroon; ${ }^{10} 25 \%$ in a village in Liberia; ${ }^{12}$ and $26 \%$ in the Madang area of Papua New Guinea). ${ }^{15}$ Nevertheless, most of the blood-feedings on gametocyte carriers in these studies did not result in the infection of mosquitoes.

In the current study, $71 \%$ of gametocyte carriers were infective for mosquitoes. This percentage is slightly higher than those found in similar studies (53\% in Burkina Faso; ${ }^{7}$ $38 \%$ or $62 \%$ in Cameroon; $8,1045 \%$ in Papua New Guinea). ${ }^{15}$ This difference may be related to the large proportion of recent malaria attacks in our gametocyte carrier sample.

Graves observed a small decline in the gametocyte infectivity after 10 minutes in the feeder, and a marked decline after 30 minutes. ${ }^{16}$ However, Ponnudurai and others who employed an improved feeder (the same one used in our study) observed that infectivity remained stable during the first 30 minutes period of feeding. ${ }^{5}$ We therefore consider our observations to be valid up to 20 minutes after blood was put into the feeder.

Acknowledgments: We would like to thank the 60 volunteers involved in the study and Samba Ngom, Moussa Diagne, Mamadou Niang, and Cheikh Yade from S.L.A.P. (Service de Lutte Anti Parasitaire au Sénégal) and, Pape Ndiaye, Paul Senghor, Louis Barboza, and El Hadj Bâ from the I.R.D. (Institut de Recherche pour le Développement, formerly Orstom) for technical help. We also thank Dr. Yves Dutheil for help at the beginning of the study, and Dr. John Beier for revision of the manuscript.
Financial support: This study was funded by the I.R.D. and by SmithKline Beecham International Africa.

Authors' addresses: Herman Parfait Awono-Ambéné, OCEAC, B.P.288 Yaoundé, Cameroun. Lamine Diawara, SLAP, B.P. A-151, Thiès, Sénégal. Vincent Robert, Institut Pasteur, B.P.1274 Antananarivo 101, Madagascar.

Reprint requests: Vincent Robert, Institut Pasteur, B.P.1274 Antananarivo 101, Madagascar; robert@pasteur.mg

\section{REFERENCES}

1. Rutledge LC, Ward RA, Gould DJ, 1964. Studies on the feeding response of mosquitoes to nutritive solutions in a new membrane feeder. Mosquito News 24: 407-419.

2. Awono-Ambene HP, Robert V, 1998. Gorging response of wild Anopheles arabiensis using membrane feeder with Baudruche or Parafilm ${ }^{\circledR}$. Parasite 5: 294.

3. Robert V, 1998. Age grading Anopheles arabiensis: their gorging and surviving responses using a membrane feeding system. Parasite 5: 87-90.

4. Robert V, Awono-Ambene HP, Thioulouse J, 1998. Ecology of larval mosquitoes, with special reference to Anopheles arabiensis (Diptera: Culicidae) in market-garden wells in urban Dakar, Senegal. J Med Entomol 35: 948-955.

5. Ponnudurai T, Lensen AHW, Van Gemert GJA, Bensink MPE, Bolmer M, Meuwissen JHET, 1989. Infectivity of cultured Plasmodium falciparum gametocytes to mosquitoes. Parasitology 98: 165-173.

6. Williams CB, 1937. The use of logarithms in the interpretation of certain entomological problems. Ann Appl Biol 24: 404-414.

7. Boudin C, Olivier M, Molez JF, Chiron JP, Ambroise-Thomas P, 1993. High human malarial infectivity to laboratory-bred Anopheles gambiae in a village in Burkina Faso. Am J Trop Med Hyg 48: 700-706.

8. Gouagna LC, Mulder B, Noubissi E, Tchuinkam T, Verhave JP, Boudin C, 1998. The early sporogonic cycle of Plasmodium falciparum in laboratory-infected Anopheles gambiae: an estimation of parasite efficacy. Trop Med Int Health 3: 21-28.

9. Robert V, Read A, Essong J, Tchuinkam T, Mulder B, Verhave JP, Carnevale P, 1996. Effect of gametocyte sex ratio on infectivity of Plasmodium falciparum to Anopheles gambiae. Trans R Soc Trop Med Hyg 90: 621-624.

10. Tchuinkam T, Mulder B, Dechering K, Verhave JP, Cot M, Carnevale P, Meuwissen J, Robert V, 1993. Experimental infections of Anopheles gambiae with Plasmodium falciparum of naturally infected gametocyte carriers in Cameroon: factors influencing the infectivity to mosquitoes. Trop Med Parasitol 44: $271-276$.

11. Githeko AK, Brandling-Bennet AD, Beier M, Atieli F, Owaga M, Collins FH, 1992. The reservoir of Plasmodium falciparum malaria in a holoendemic area of western Kenya. Trans $R$ Soc Trop Med Hyg 86: 355-358.

12. Muirhead-Thompson RC, 1957. The malaria infectivity of an African village population to mosquitoes (An. gambiae). A random xenodiagnostic survey. Am J Trop Med Hyg 6: 971-979.

13. Robert V, Le Goff G, Gouagna LC, Sinden M, Kieboom J, Kroneman R, Verhave JP, 1998. Kinetics and efficiency of Plasmodium falciparum development in the midguts of Anopheles gambiae, An. funestus and An. nili. Ann Trop Med Parasitol 92: $115-118$.

14. Rutledge LC, Hayes DE, Waird RA, 1970. Plasmodium cynomolgi: sources of variation in susceptibility of Anopheles quadrimaculatus, An. balabacensis and An. stephensi. Exp Parasitol 27: 53-59.

15. Graves PM, Burkot TR, Carter R, Cattan JA, Lagog M, Parker J, Brabin BJ, Gibson FD, Bradley DJ, Alpers MP, 1988. Measurement of malarial infectivity of human populations to mosquitoes in the Madan area, Papua New Guinea. Parasitology 96: 251-263.

16. Graves PM, 1980. Studies on the use of a membrane feeding technique for infecting Anopheles gambiae with Plasmodium falciparum. Trans R Soc Trop Med Hyg 74: 738-742. 